\title{
Avaliação do Desempenho de Catalisadores Híbridos Ziegler-Natta/Metaloceno na Homo- e Copolimerização de Etileno
}

\author{
Fernanda 0. V. Cunha e Madalena C. Forte \\ Laboratório de Materiais Poliméricos, Departamento de Materiais, Escola de Engenharia, UFRGS
}

Jorge J . Zacca
Centro de Tecnologia - OPP Petroquímica

\begin{abstract}
Resumo: Foram preparados catalisadores híbridos a partir de um catalisador do tipo Ziegler-Natta heterogêneo $\left(\mathrm{MgCl}_{2}\right.$. TiCl $)$ e de um metalocênico $\left(\mathrm{CpTiCl}_{3}\right)$, afim de se obter catalisadores com diferentes tipos de centros ativos. Foi proposta a formação de um novo centro ativo $\left(\mathrm{CpTi}(\mathrm{iBu}) \mathrm{Cl}_{2}\right)$ na face 100 do suporte de $\mathrm{MgCl}_{2}$. Estes catalisadores foram avaliados na síntese de polietileno de alta densidade (PEAD) e de polietileno linear de baixa densidade (PELBD), utilizando-se somente trietilalumínio (TEA) como cocatalisador. Foi verificada a influência da razão molar $\mathrm{Al} / \mathrm{Ti}$, temperatura e tempo de polimerização na produtividade dos catalisadores e nas propriedades dos polímeros obtidos. Os catalisadores mistos apresentaram atividade catalítica da ordem de $5,0 \mathrm{kgPol} / \mathrm{g}_{\text {cat }}$.h na polimerização de etileno e da ordem de $7,0 \mathrm{kgPol} / \mathrm{g}_{\text {cat }}$. $\mathrm{h}$ na copolimerização etileno-1-buteno.
\end{abstract}

Palavras-chave: Catalisador híbrido, PEAD, PELBD, catalisador Ziegler-Natta, catalisador metalocênico.

\section{The Performance Evaluation of Hybrid Ziegler-Natta/Metallocene Catalysts in Homo- and Copolymerization of Ethylene}

Abstract: Hybrid catalysts formed by a heterogeneous Ziegler-Natta catalyst $\left(\mathrm{MgCl}_{2} \cdot \mathrm{TiCl}_{4}\right)$ and a metallocene $\left(\mathrm{CpTiCl}_{3}\right)$ were prepared in this work. The formation of a new catalytic species $\mathrm{CpTi}(\mathrm{iBu}) \mathrm{Cl}_{2}$ on the $(100)$ $\mathrm{MgCl}_{2}$ face has been proposed. These catalyst systems were applied to synthesize high density polyethylene (HDPE) and linear low density polyethylene (LLDPE), using only triethyl aluminium (TEA) as a cocatalyst. The influence of Al/Ti molar ratio, temperature and time of polymerization on the catalyst activity and on the polymer properties were evaluated. The catalyst activities were about $5.0 \mathrm{kgPol} / \mathrm{g}_{\mathrm{cat}} \cdot \mathrm{h}$ for ethylene polymerization and $7.0 \mathrm{kgPol} / \mathrm{g}_{\text {cat }}$ h for ethylene-1-butene coplymerization.

Keywords: Hybrid catalyst, HDPE, LLDPE, Ziegler-Natta catalyst, metallocene catalyst.

\section{Introdução}

Nas últimas décadas vêm se aprimorando cada vez mais os sistemas catalíticos para a polimerização de olefinas, quer seja através da modificação dos catalisadores atuais ou pelo desenvolvimento de novos sistemas ativos. A partir dos trabalhos pioneiros de Kaminsky/Sinn ${ }^{[1-2]}$ e Ewen ${ }^{[3]}$ sobre compostos metalocênicos, cocatalisados por metilaluminoxano (MAO), foi desenvolvida uma nova família de catalisadores capazes de produzir polímeros com propriedades especiais com alto rendimento.

Os sistemas catalíticos metalocênicos são essencialmente homogêneos e a sua heterogeinização represen-

Autor para correspondência: Madalena C. Forte, Laboratório de Materiais Poliméricos, Departamento de Materiais - Escola de Engenharia, UFRGS, Av. Osvaldo Aranha 99, CEP: 90035-190, Porto Alegre, RS. E-mail: mmcforte@ufrgs.br 
ta um desafio a ser solucionado em nível industrial, uma vez que os processos de polimerização de última geração utilizam catalisadores heterogêneos. Além disso, as poliolefinas produzidas por esses sistemas catalíticos, os quais apresentam um único tipo de centro ativo, possuem distribuição de pesos moleculares estreita, fato este que dificulta a processabilidade do polímero, o que também deverá ser solucionado. ${ }^{[4,5]}$ Os catalisadores metalocênicos, ao contrário dos catalisadores Ziegler-Natta convencionais, são capazes de produzir resinas etilênicas com propriedades diferenciadas, devido ao fato de que na produção de polietileno linear de baixa densidade (PELBD) a incorporação e a distribuição do comonômero na cadeia polimérica é homogênea ${ }^{[6]}$. Os PELBD produzidos pela catálise convencional, além de não possuírem distribuição homogênea do comonômero na cadeia, apresentam moléculas de menor massa molecular com maior grau de incorporação desse.

Os catalisadores Ziegler-Natta heterogêneos utilizados na polimerização de etileno, quer seja na produção de polietileno de alta densidade (PEAD) ou de baixa densidade (PELBD), são quase que exclusivamente à base de tetracloreto de titânio $\left(\mathrm{TiCl}_{4}\right)$ suportado em cloreto de magnésio $\left(\mathrm{MgCl}_{2}\right)$. Este último é geralmente tratado com doadores de elétrons, normalmente ftalato de diisobutila (doadores de elétrons internos - DI), durante a preparação do catalisador.

O objetivo deste trabalho foi preparar catalisadores híbridos que apresentassem características de ambos os catalisadores, Ziegler-Natta convencional e metalocênico, e avaliar o desempenho destes na homo e copolimerização de etileno e 1-buteno, utilizando somente trietilalumínio (TEA) como cocatalisador. Os catalisadores mistos foram preparados pela fixação de um composto metalocênico $\left(\mathrm{CpTiCl}_{3}\right)$ em um catalisador Ziegler-Natta heterogêneo do tipo $\mathrm{MgCl}_{2}$.DI.TiCl 4 .

\section{Experimental}

Os catalisadores Ziegler-Natta convencionais e metalocênicos utilizados neste trabalho foram adquiridos respectivamente da Akzo Nobel Chemicals Inc. e Boulder Scientific Company e usados como recebidos. Os cocatalisadores triisobutilalumínio (TIBA), utilizado na síntese do catalisador, e o trietilalumínio (TEA), usado na polimerização, foram adquiridos da Akzo Nobel Chemicals Inc. e usados em solução de n-hexano. O n-hexano grau polímero (IBRASOL), utilizado na preparação dos catalisadores e na polimerização, foi armazenado em peneira molecular $3 \AA$. O manuseio dos componentes catalíticos e as manipulações na preparação dos catalisadores e polimerizações foram realizados sob atmosfera de $\mathrm{N}_{2}$ utilizando-se a técnica de Schlenk ${ }^{[7]}$.

\section{Preparação dos catalisadores}

Em um tubo Schlenk de $20 \mathrm{ml}$ foram adicionados $218 \mathrm{mg}$ do composto metalocênico $\mathrm{CpTiCl}_{3}$ sólido (1mmol) e $8 \mathrm{ml}$ de $\mathrm{n}$-hexano. Em seguida, sob agitação magnética, foi adicionado lentamente à suspensão $0,7 \mathrm{ml}$ de TIBA $100 \%$ ( $2.8 \mathrm{mmol})$. A mistura permaneceu sob agitação durante 2 horas à temperatura ambiente até a obtenção de uma solução de coloração preta. Esta solução foi então adicionada a uma suspensão do catalisador Ziegler-Natta ( 2 mmol de Ti em 80 $\mathrm{ml}$ de $\mathrm{n}$-hexano), em reator de vidro sinterizado equipado com agitador mecânico. A suspensão foi mantida sob agitação a $300 \mathrm{rpm}$ durante 5 horas a $40^{\circ} \mathrm{C}$. Em seguida o sólido residual foi lavado com hexano e seco em leito fluidizado de nitrogênio. Foram preparados catalisadores com razão molar de titânio ZieglerNatta(ZN)/titânio metaloceno (ZN/Met) de 2 a 5.

Os catalisadores foram caracterizados por meio de análises de $\mathrm{Ti}, \mathrm{Cl}$ e $\mathrm{Mg}$ por via úmida, conforme métodos relatados da literatura ${ }^{[8]}$ e análise de espectrometria fotoelétrica de raios-X (XPS).

\section{Polimerizações de etileno}

As polimerizações de etileno para obtenção de polietileno de alta densidade (PEAD) foram feitas em reator tipo autoclave de 4 litros equipado com agitação mecânica e controle de temperatura. As reações de polimerização em suspensão de hexano (2 L) foram realizadas a $400 \mathrm{rpm}$, pressão total de 7 bar em temperaturas de 75 e $85^{\circ} \mathrm{C}$. Foram realizados estudos variando-se a razão $\mathrm{Al} / \mathrm{Ti}$, temperatura e tempo de reação, e quantidade de hidrogênio adicionada ao sistema. A ordem de adição dos componentes no reator foi a seguinte: solvente, cocatalisador (TEA), catalisador, hidrogênio e etileno. $\mathrm{O}$ catalisador utilizado na homopolimerização foi aquele preparado com a razão molar ZN/Met igual a 2 .

\section{Copolimerizações de etileno/1-buteno}

As copolimerizações para a obtenção de polietileno linear de baixa densidade (PELBD) foram feitas conforme a descrita para a polimerização de etileno, uti- 
lizando-se como comonômero o 1-buteno, temperaturas de 75 e $90{ }^{\circ} \mathrm{C}$ e tempo de reação de $1 \mathrm{~h}$. Em todas as polimerizações a quantidade de hidrogênio foi sempre a mesma, igual a $0,36 \mathrm{~g}$ e a pressão total de reação foi de 9 bar. Foram variados a razão Al/Ti e o tipo de catalisador, segundo a razão molar de titânio ZN/Met. A ordem de adição dos componentes foi a seguinte: solvente, cocatalisador (TEA), catalisador, 1-buteno, hidrogênio e por último o etileno.

\section{Caracterização dos polímeros}

O índice de fluidez dos polímeros foi determinado em um plastômetro Tinius Olsen MP 987 utilizando-se o peso de $2,16 \mathrm{~kg}$ e temperatura de $190{ }^{\circ} \mathrm{C}$.

Os copolímeros foram caracterizados quanto ao teor de comonômero incorporado em amostras sob forma de filme com espessura em torno de $20 \mu \mathrm{m}$, por FT-IR em equipamento Nicolet 710, utilizandose as bandas com comprimento de onda de 4482 a $3950 \mathrm{~cm}^{-1}$ relacionadas com a espessura do filme e a banda de deformação do grupo metila a $1378 \mathrm{~cm}^{-1[9]}$.

\section{Resultados e Discussão}

Os catalisadores à base de $\mathrm{TiCl}_{4}$ suportado em $\mathrm{MgCl}_{2}$ apresentam de uma maneira geral dois tipos principais de centros ativos, dependendo da acidez ou basicidade da face do suporte onde se encontram fixados. Os centros isoespecíficos formados pela espécie bimolecular $\left(\mathrm{Ti}_{2} \mathrm{Cl}_{6}\right)$ nas faces menos ácidas (100) e (101), e os centros não específicos $\left(\mathrm{TiCl}_{3}\right)$, na face mais ácida do $\mathrm{MgCl}_{2}$ (110), são do tipo daqueles mostrados na Figura 1 (b) e (c) ${ }^{[10-13]}$. Estes centros são formados pela fixação de $\mathrm{TiCl}_{4}$ sobre as faces do $\mathrm{MgCl}_{2}$ tratado com um doador de elétrons interno, o qual é responsável pela alta estereoespecificidade do catalisador $^{[14]}$. A vizinhança química do centro isoespecífico determina a atividade do catalisador e tamanho da cadeia a ser produzida, isto é, ambas dependem da localização do centro ativo no suporte, da proximidade deste do doador de elétrons interno e da interação com o complexo alquilalumínio - doador de elétrons externo, durante a polimerização.

Para a obtenção de um catalisador híbrido foi introduzida uma nova espécie catalítica à base de titânio no catalisador heterogêneo contendo substituintes diferentes de cloro, por exemplo, o $\mathrm{CpTiCl}_{3}$, que poderia, portanto, modificar a química do catalisador.

A fixação do composto metalocênico no catalisador Ziegler-Natta sólido de partida foi executada em duas etapas: a) solubilização do composto metalocênico sólido em hexano, pelo tratamento deste com uma solução de triisobutilalumínio na proporção molar de Al/Ti igual a 2,8; b) contato da solução contendo o composto metalocênico com o catalisador Ziegler-Natta sólido, em diferentes razões molares de titânio do catalisador Ziegler-Natta para o de titânio do composto metalocênico. No tratamento do catalisador metalocênico com o triisobutilalumínio, ocorre a alquilação deste, com a formação do composto $\mathrm{CpTi}(\mathrm{iBu}) \mathrm{Cl}_{2}$ o qual, com a introdução do grupo isobutila passa a ser solúvel no meio. A fixação do composto $\mathrm{CpTi}(\mathrm{iBu}) \mathrm{Cl}_{2}$ com titânio ligado a um anel ciclopentadienila introduz um novo tipo de centro ativo no catalisador convencional conforme proposto neste trabalho e mostrado na Figura 1 (c). Esta nova espécie catalítica se fixa na face (100) do cloreto de magnésio através de dois cloros, na forma monomolecular, já apresentando um grupo alquila e uma vacância. A fixação desta espécie na face (110) não leva à formação de espécies ativas devido à inexistência de cloro livre para a formação de uma vacância.

A Tabela 1 mostra os resultados das análises de titânio, cloro e magnésio para alguns catalisadores híbridos preparados a partir de um catalisador Ziegler-

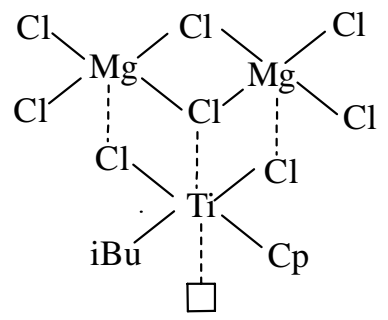

(a)

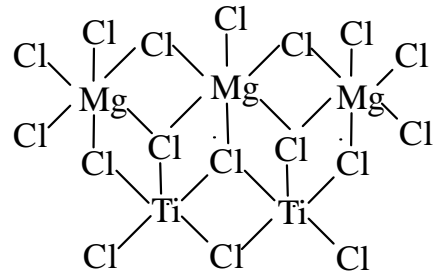

(b)

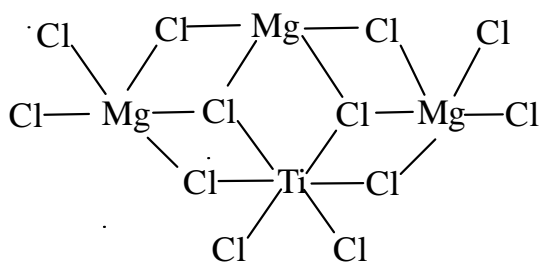

(c)

Figura 1. Tipos de centros ativos presentes no catalisador Ziegler-Natta. a) centro proposto neste trabalho para o composto $\mathrm{CpTi}(\mathrm{iBu}) \mathrm{Cl}_{2}$ suportado na face (100) do $\mathrm{MgCl}_{2}$, b) centro isoespecíficos na face (100) do $\mathrm{MgCl}_{2} \mathrm{e}$ c) centro não específico na face (110) do $\mathrm{MgCl}_{2}^{[13]}$ 
Tabela 1. Teores de $\mathrm{Ti}, \mathrm{Cl}$ e $\mathrm{Mg}$ de catalisadores mistos Ziegler-Natta/ Metaloceno com diferentes razões molares de titânio ZN/Met. ${ }^{\text {a) }}$

\begin{tabular}{lccccc}
\hline Catalisador & ZN/Met. & \% Ti & \% Cl & \% Mg & Outros $^{\text {b) }}$ \\
\hline ZNM 20 & 2 & 3,2 & 57,5 & 17,3 & 22,0 \\
ZNM 04 & 3 & 2,9 & 58,8 & 15,9 & 22,4 \\
ZNM 03 & 5 & 2,4 & 55,3 & 16,4 & 25,9 \\
Ziegler-Natta & & 2,2 & 58,6 & 18,7 & $20,5^{\text {c) }}$ \\
\hline
\end{tabular}

a) Determinação por métodos de análise de via úmida. Valor médio de três determinações

b) Alumínio e orgânicos (doador de elétrons interno, alquilalumínio, metaloceno e hexano)

c) Orgânicos (doador de elétrons interno e hexano)

Natta comercial, usado como catalisador base, e o composto metalocênico $\mathrm{CpTiCl}_{3}$.

Em todos os catalisadores preparados notou-se que o teor de titânio foi sempre superior ao do catalisador base e este aumentava com o acréscimo da quantidade de metaloceno na mistura reacional, na preparação do catalisador misto. Tal variação sugere que, de uma maneira geral, o catalisador metalocênico se fixa no catalisador base, mais provavelmente no suporte de cloreto de magnésio, devido à maior diferença de acidez de Lewis entre estes. A fixação do composto $\mathrm{CpTi}(\mathrm{iBu}) \mathrm{Cl}_{2}$ possibilita assim a formação de uma nova espécie ativa para a polimerização de olefinas. O percentual de cloretos dos catalisadores preparados foi de $58 \pm 3,0 \%$ e o de magnésio de $16 \pm 1,6 \%$, sendo o intervalo de variação o desvio da média aritmética dos valores encontrados. Esta variação pode ser atribuída à maior ou menor quantidade do catalisador metalocênico fixado, ou do cocatalisador, e à eventual remoção do doador de elétrons interno, bem como ao erro inerente à análise. Na Tabela 1 , o valor atribuído a outros se refere a materiais orgânicos e alumínio que estão presentes no catalisador, sendo os orgânicos o doador de elétrons interno, o grupo ciclopentadienila do composto metalocênico e o grupo isobutila do alquilalumínio, estando este último também fixado no catalisador.

A técnica de XPS permite identificar o tipo de elemento presente na superfície do catalisador bem como o estado de oxidação do mesmo ${ }^{[15-18]}$. Neste caso foi investigado o tipo de titânio presente no catalisador Ziegler-Natta base e nos catalisadores tratados com metaloceno (ZNM 21 e ZNM24), Figura 2.

Os espectros dos catalisadores ZNM 21 e ZNM 24 apresentaram dois picos na região $2 \mathrm{p}_{3 / 2}$ referentes a dois tipos de titânio, enquanto que o catalisador
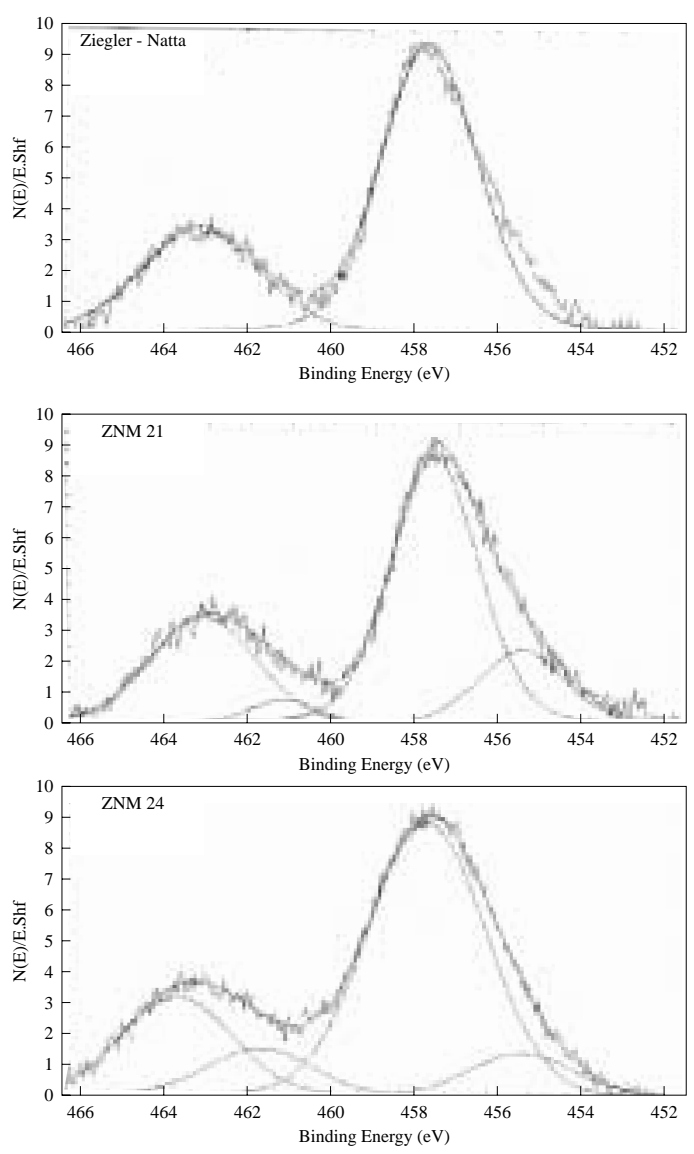

Figura 2. Análise de XPS de Ti do catalisador Ziegler-Natta comercial e dos catalisadores hibridos.

Ziegler-Natta apresenta apenas o pico, nesta região, referente ao titânio no estado de oxidação IV. O pico na região de menor energia pode ser atribuído a presença do Ti do catalisador metalocênico.

A avaliação do catalisador misto na homopolimerização de etileno foi feita com o catalisador preparado com razão molar de titânio ZN/Met igual a 2 $(\mathrm{ZN} / \mathrm{Met}=2)$. Neste estudo foi variada a razão Al/Ti, o tempo e a temperatura de reação, tendo-se como parâmetro de avaliação a atividade catalítica na produção de PEAD, expressa em kilograma de polietileno por grama de catalisador por hora $\left(\mathrm{kgPE} / \mathrm{g}_{\text {cat }} \cdot \mathrm{h}\right)$. A Tabela 2 mostra os valores de atividade catalítica obtidos para diferentes valores de razão molar Al/Ti, temperatura e tempo de reação, em polimerizações de etileno em n-hexano, utilizando-se como cocatalisador somente trietilalumínio.

Os valores de atividade catalítica, expressos para uma hora de reação, em função da razão molar Al/Ti utilizada na polimerização de etileno, de 500 a 1100 , estão dentro do intervalo de variação normalmente observado para catálise heterogênea que é de $20 \%$. Tal 
Cunha, F. 0. V. et al. - Desempenho de catalisadores Ziegler-Natta/metaloceno na polimerização de etileno

Tabela 2. Influência da razão molar Al/Ti, da temperatura e do tempo de polimerização na atividade do catalisador híbrido ZN/Met $=2,0$ na produção de PEAD.

\begin{tabular}{|c|c|c|c|c|c|}
\hline Teste N. ${ }^{\circ}$ & $\begin{array}{c}\mathrm{Al} / \mathrm{Ti} \\
\text { (razão molar) }\end{array}$ & $\begin{array}{l}\text { Temperatura } \\
\left({ }^{\circ} \mathrm{C}\right)\end{array}$ & $\begin{array}{c}\text { Tempo } \\
\text { (h) }\end{array}$ & $\begin{array}{l}\text { Atividade } \\
\text { Por reação }\end{array}$ & $\begin{array}{l}\left(\mathrm{kgPE} / \mathrm{g}_{\text {cat }} . \mathbf{h}\right) \\
\text { média aritmética }\end{array}$ \\
\hline 1 & 100 & 75 & 1 & $\mathrm{np}$ & $\mathrm{np}$ \\
\hline 2 & 500 & 75 & 1 & 3,7 & \\
\hline 3 & 500 & 75 & 1 & 4,8 & $4,3 \pm 0,6$ \\
\hline 4 & 500 & 75 & 1 & 5,1 & \\
\hline 5 & 800 & 75 & 1 & 4,4 & \\
\hline 6 & 800 & 75 & 1 & 5,6 & $5,0 \pm 0,6$ \\
\hline 7 & 800 & 75 & 1 & 5,2 & \\
\hline 8 & 950 & 75 & 1 & 5,0 & 5,0 \\
\hline 9 & 1100 & 75 & 1 & 6,4 & \\
\hline 10 & 1100 & 75 & 1 & 5,7 & $6,0 \pm 0,4$ \\
\hline 11 & 1100 & 75 & 2 & 4,8 & \\
\hline 12 & 1100 & 75 & 2 & 5,2 & $5,0 \pm 0,2$ \\
\hline 13 & 1100 & 75 & 3 & 5,0 & 5,0 \\
\hline 14 & 1100 & 75 & 4 & 5,1 & 5,1 \\
\hline 15 & 1100 & 85 & 1 & 5,5 & \\
\hline 16 & 1100 & 85 & 1 & 5,4 & $5,4 \pm 0,2$ \\
\hline 16 & 1100 & 85 & 2 & 5,1 & \\
\hline 17 & 1100 & 85 & 2 & 5,7 & $5,4 \pm 0,3$ \\
\hline 18 & 1100 & 85 & 3 & 6,2 & \\
\hline 19 & 1100 & 85 & 3 & 6,2 & $6,2 \pm 0,2$ \\
\hline 20 & 1500 & 75 & 1 & 4,0 & \\
\hline 21 & 1500 & 75 & 1 & 5,3 & $4,6 \pm 0,6$ \\
\hline 22 & 1500 & 75 & 1 & 4,5 & \\
\hline
\end{tabular}

Condições de polimerização: Reator tipo autoclave $4 \mathrm{~L}, \mathrm{n}$-hexano $=2 \mathrm{~L} ;$ [cat.] $=1,3 \mathrm{mmol}$; cocatalisador $=$ TEA; $\mathrm{P}_{\text {eteno }}=7$ bar; $\mathrm{np}=$ não polimeriza

comportamento é atípico, uma vez que com catalisadores Ziegler-Natta, normalmente ocorre um acréscimo significativo da atividade, maior que $20 \%$, com o aumento da razão molar $\mathrm{Al} / \mathrm{Ti}$ até um certo limite ou patamar de atividade. Em valores de razão molar $\mathrm{Al} / \mathrm{Ti}$ muito elevados ocorre uma diminuição da atividade devido a uma super redução do metal de transição. Em valores de razão Al/Ti muito baixos a atividade normalmente é baixa devido a não ativação dos centros ativos ou desativação destes por impurezas no meio. Tal resultado pode ser atribuído ao fato do catalisador já ter tido um contato prévio com alquilalimínio, ou ter sido quimicamente modificado por este durante sua preparação. Essa característica possibilita a utilização de baixas concentrações de alquilalumínio na polimerização, o que leva a uma potencial redução de custos e de contaminação do produto final por alumínio. Para valores de razão molar Al/ Ti inferiores a 500 não se observou atividade catalítica na polimerização de etileno.

A elevação da temperatura de polimerização de 75 para $85^{\circ} \mathrm{C}$, para o tempo de reação de 1 hora, não teve influência significativa na atividade do catalisador na produção de PEAD. Dentro do intervalo de tempo de reação testado de até 4 horas, observou-se um aumento linear da produtividade do catalisador com o tempo de polimerização, permanecendo a atividade catalítica constante neste intervalo. Tal fato torna o catalisador bastante atrativo para a produção de PEAD com distri- 
buição de pesos moleculares bimodal ou larga por meio da utilização de um processo de polimerização em dois reatores em série. Neste tipo de processo, os catalisadores promissores são os que possuem uma atividade catalítica elevada durante um período no mínimo de 2 horas. O catalisador híbrido preparado neste trabalho é potencialmente ativo, uma vez que apresenta atividade constante ou significativa num período de até 4 horas. Este tempo é superior ao de residência do catalisador no primeiro reator, em torno de 1 hora, e portanto o catalisador ainda será efetivo para a produção de resina no segundo reator. A distribuição de peso molecular larga ou bimodal se faz pelo controle do peso molecular da resina em ambos os reatores pela adição diferenciada de hidrogênio ${ }^{[19,20]}$.

Para avaliar a capacidade do catalisador frente às reações de transferência de cadeia com hidrogênio foram feitas reações de polimerização em três concentrações de $\mathrm{H}_{2}$ na faixa de 1,0 a $1,6 \mathrm{~g}$ pelo volume total do reator $(4 \mathrm{~L})$. Nessas reações foi possível obter-se polímeros com peso molecular adequados para a realização de análises de índice de fluidez (IF). A Tabela 3 apresenta os resultados obtidos de atividade catalítica e índice de fluidez dos polímeros em função da concentração de hidrogênio no meio de polimerização. Para as concentrações testadas a atividade não apresentou variação significativa, entretanto, o índice de fluidez dos polímeros variou significativamente. Essa variação demonstra uma boa sensibilidade do catalisador à transferência de cadeia com hidrogênio e consequiente controle do peso molecular. Os valores de IF obtidos para os polímeros, nas condições utilizadas neste trabalho, são da ordem
Tabela 3. Influência da concentração de hidrogênio na atividade do catalisador híbrido Ziegler-Natta/Metaloceno e no índice de fluidez dos polímeros.

\begin{tabular}{cccc}
\hline${\text { Teste } \mathbf{N}^{\mathbf{0}}}$ & $\mathbf{C H}_{\mathbf{2}}(\mathbf{g} / \mathbf{4 L})$ & $\begin{array}{c}\text { Atividade } \\
\left(\mathbf{k g P E} / \mathbf{g}_{\text {cat }} \cdot \mathbf{h}\right)\end{array}$ & $\begin{array}{c}\mathbf{I F}^{\text {a) }} \\
(\mathbf{g} / \mathbf{1 0} \mathbf{~ m i n})\end{array}$ \\
\hline 24 & 1,0 & 5,7 & 0,11 \\
25 & 1,2 & 4,6 & 0,26 \\
26 & 1,6 & 5,2 & 0,46
\end{tabular}

Condições de polimerização: Reator tipo autoclave $4 \mathrm{~L}, \mathrm{n}$-hexano $=2 \mathrm{~L}$, catalisador $\mathrm{ZN} / \mathrm{Met}=2$, [cat.] $=1,3 \mathrm{mmol}, \mathrm{Al} / \mathrm{Ti}=1100, \mathrm{P}_{\text {total }}=7 \mathrm{bar}, \mathrm{t}=2 \mathrm{~h}$. a) Índice de fluidez (peso $=2,16 \mathrm{~kg} ; \mathrm{T}=190^{\circ} \mathrm{C}$ ).

de grandeza daqueles do PEAD comercial, ou seja de 0,1 a $1,5 \mathrm{~g} / 10 \mathrm{~min}$.

Afim de se avaliar o desempenho do catalisador misto também em copolimerizações de etileno e alfa-olefinas, foram obtidos PELBD utilizando-se 1-buteno como comonômero. Foram utilizados os catalisadores mistos preparados com diferentes razões molares de titânio ZN/ Met. Foram avaliadas também a influência da razão mo$\operatorname{lar} \mathrm{Al} / \mathrm{Ti}$ e a concentração de 1-buteno na atividade do catalisador e no teor de comonômero incorporado.

A Tabela 4 mostra os valores de atividade catalítica (KgPELBD $/ \mathrm{g}_{\text {cat. }} \mathrm{h}$ ), teor de comonômero incorporado e IF dos copolímeros obtidos em função da razão molar Al/Ti, e da temperatura de polimerização. O catalisador híbrido na copolimerização de etileno-1buteno, diferentemente da homopolimerização de etileno, apresenta atividade catalítica em valores de razão molar $\mathrm{Al} / \mathrm{Ti}$ da ordem de 60 , inferior a mínima observada no primeiro caso, ou seja da ordem de 500.

Para razão molar Al/Ti igual a 60, conforme já esperado, não houve formação de polímero com o catalisador Ziegler-Natta, uma vez que neste caso há

Tabela 4. Influência da razão molar Al/Ti e temperatura de reação, em copolimerização de etileno e 1-buteno, na atividade catalítica, teor de comonômero incorporado e índice de fluidez dos copolímeros.

\begin{tabular}{|c|c|c|c|c|c|c|}
\hline Teste $\mathbf{N}^{\circ}$ & Catalisador & $\begin{array}{c}\mathbf{A l} / \mathbf{T i} \\
\text { (razão molar) }\end{array}$ & $\begin{array}{l}\text { Temp. } \\
\left({ }^{\circ} \mathrm{C}\right)\end{array}$ & $\begin{array}{c}\text { Atividade } \\
\left(\mathrm{kgPELBD} / \mathrm{g}_{\text {cat }}, \mathrm{h}\right)\end{array}$ & $\begin{array}{c}\text { Teor de } \mathrm{C}_{4} \\
\text { incorp. }\left(\% \text { peso) }{ }^{\mathrm{b})}\right.\end{array}$ & $\begin{array}{c}\mathrm{IF}^{\mathrm{c})} \\
(\mathrm{g} / \mathbf{1 0 \mathrm { min }})\end{array}$ \\
\hline 34 & Ziegler Natta & 60 & 75 & $\mathrm{np}$ & - & - \\
\hline 35 & Ziegler Natta & 60 & 90 & $\mathrm{np}$ & - & - \\
\hline 36 & Ziegler-Natta & 600 & 75 & 5.1 & 5.5 & 0.60 \\
\hline 37 & Ziegler-Natta & 600 & 90 & 7.5 & 6.2 & 0.88 \\
\hline 38 & Hídrido ${ }^{\text {a) }}$ & 60 & 75 & 6.9 & 2.4 & 0.01 \\
\hline 39 & Hídrido a) & 60 & 90 & 0.4 & 7.8 & 0.01 \\
\hline 40 & Hídrido a) & 600 & 75 & 11.7 & 4.1 & 0.01 \\
\hline 41 & Hídrido a) & 600 & 90 & 4.4 & 7.7 & 0.30 \\
\hline
\end{tabular}

Condições de polimerização: Reator autoclave $4 \mathrm{~L}, \mathrm{n}$-hexano $=2 \mathrm{~L}$, [cat.] $=3,5 \mathrm{mmol}, \mathrm{C}_{\text {hidrogênio }}=0,36 \mathrm{~g} / 4 \mathrm{~L}, \mathrm{P}_{\text {total }}=9 \mathrm{bar}, \mathrm{t}=1 \mathrm{~h}$. $\mathrm{np}=\mathrm{não}$ polimeriza. a) catalisador ZN/Met. = 2. b) Determinado por FT-IR, c) Índice de fluidez (peso = 2,16kg; $\mathrm{T}=190^{\circ} \mathrm{C}$ ). 
necessidade de uma concentração mínima do cocatalisador para eliminação de impurezas do meio, redução e alquilação do Ti do catalisador. Quando comparado ao catalisador Ziegler-Natta convencional observa-se que o catalisador hídrido é capaz de polimerizar nestes baixos valores de razão molar Al/Ti e na temperatura de $75^{\circ} \mathrm{C}$ este é significativamente mais ativo. $\mathrm{O}$ fato importante neste caso, constatado experimentalmente, é que o catalisador hídrido é capaz de copolimerizar em concentrações mais baixas de alumínio do que o catalisador Ziegler-Natta convencional, o que pode ser atribuído ao fato de que o primeiro, devido à presença de TIBA na sua preparação, tenha sido pré-reduzido, e assim necessite de menor quantidade de cocatalisador para a sua ativação.

O aumento da temperatura de 75 para $90{ }^{\circ} \mathrm{C}$ leva a uma diminuição na atividade do catalisador híbrido, diferente do que foi observado na obtenção de PEAD, onde a atividade não foi afetada pela variação de temperatura. Com o catalisador Ziegler-Natta convencional nota-se o efeito contrário, o aumento da temperatura provocou um aumento na atividade catalítica conforme o usualmente observado. Tal fato poderia ser atribuído a uma desativação dos sítios catalíticos metalocênicos do catalisador híbrido em temperaturas mais elevadas, corroborando o comportamento observado quando o catalisador $\mathrm{CpTiCl}_{3}$ é utilizado em polimerização homogênea $^{[21]}$. Por outro lado, a elevação da temperatura provocou um aumento na incorporação de comonômero na cadeia polimérica da mesma ordem de grandeza para ambos os catalisadores Ziegler-Natta e misto. Esse comportamento pode ser atribuído ao favorecimento da reação de inserção da alfa-olefina (1- buteno).

A Tabela 5 mostra os valores da atividade catalítica e do teor de comonômero incorporado em função da razão molar de titânio ZN/Met no catalisador hídrido.
O tipo de catalisador hídrido, ou seja a razão molar de titânio ZN/Met, não tem influência na atividade do sistema catalítico, permanecendo em torno de $7,0 \mathrm{kgPELBD} / \mathrm{g}_{\mathrm{cat}} \cdot \mathrm{h}$, a variação em torno deste é inferior a 20\%, limite este aceitável para polimerizações com catálise heterogênea. Normalmente em reações de copolimerização se observa um aumento da atividade do catalisador pela adição do comonômero no meio reacional com relação à homopolimerização. Tal fato justifica a produção de polímero com o catalisador hídrido a baixas concentrações de alquilalumínio no meio reacional, da ordem de razão molar Al/Ti igual a 60. Na homopolimerização de etileno para razões molares Al/Ti menores que 500 não se observou atividade para o catalisador hídrido com razão molar de titânio ZN/Met igual a 2.

Com relação à incorporação de comonômero na cadeia polimérica, para os catalisadores hídrido testados, esta variou de 2,4 a 7,0\% não se observando nenhuma tendência entre o teor de comonômero incorporado e a razão ZN/Met do catalisador. Entretanto os catalisadores com razão ZN/Met $=2$ apresentaram maior grau de incorporação de comonômero. Os sítios catalíticos do tipo metaloceno de uma maneira geral favorecem a entrada da alfa olefina na cadeia polimérica. Assim pode-se concluir que quanto maior a quantidade deste tipo de centro catalítico ativo maior será o teor de 1-buteno incorporado no polímero. Para um aumento da concentração de 1-buteno no meio reacional de 29 a 44 g/l (massa de comonômero por volume da fase líquida) não se observou aumento da atividade catalítica, mas somente variação no teor de comonômero incorporado e índice de fluídez dos copolímeros obtidos.

Conforme já esperado, o aumento da concentração de comonômero no meio reacional levou a um

Tabela 5. Avaliação da influência do tipo de catalisador híbrido e da concentração de 1-buteno no meio reacional na atividade catalítica, no teor de comonômero incorporado e no índice de fluídez.

\begin{tabular}{|c|c|c|c|c|c|}
\hline Teste $\mathbf{N}^{\circ}$ & $\begin{array}{c}\text { Razão molar } \\
\text { Zn/Met. }\end{array}$ & $\begin{array}{c}\text { Concentração de } C_{4} \\
(\mathrm{~g} / \mathrm{L})\end{array}$ & $\begin{array}{c}\text { Atividade } \\
\left(\mathrm{kg} \mathrm{PELBD} / \mathrm{g}_{\text {cat }} . \mathrm{h}\right)\end{array}$ & $\begin{array}{c}\text { Teor de } \mathrm{C}_{4} \text { Incorp. } \\
(\% \text { peso })^{\text {a) }}\end{array}$ & $\begin{array}{c}\mathrm{IF}^{\mathrm{b})} \\
(\mathrm{g} / \mathbf{1 0} \mathrm{min})\end{array}$ \\
\hline 27 & 1 & 29,5 & 6,8 & 7,0 & nd \\
\hline 28 & 2 & 29,5 & 6,8 & 2,4 & nd \\
\hline 29 & 2 & 29,5 & 8,0 & 3,1 & nd \\
\hline 30 & 5 & 29,5 & 7,3 & 4,9 & 0,33 \\
\hline 31 & 5 & 36,9 & 4,3 & 5,2 & 0,83 \\
\hline 32 & 5 & 44,2 & 6,2 & 11,5 & 4,10 \\
\hline
\end{tabular}

Condições de polimerização: Reator autoclave $4 \mathrm{~L}, \mathrm{n}$-hexano $=2 \mathrm{~L}$, [cat.] $=3,5 \mathrm{mmol}, \mathrm{Al} / \mathrm{Ti}=60, \mathrm{~T}=75^{\circ} \mathrm{C}, \mathrm{C}_{\text {hidrogênio }}=0,36 \mathrm{~g} / 4 \mathrm{~L}, \mathrm{P}_{\text {total }}=9 \mathrm{bar}$, $\mathrm{t}=1 \mathrm{~h}$. $\mathrm{nd}=$ não determinado. a) Determinado por FT-IR. b) Índice de fluidez (peso $=2,16 \mathrm{~kg} ; \mathrm{T}=190^{\circ} \mathrm{C}$ ). 
aumento do teor de 1-buteno incorporado, sendo que um aumento de $50 \%$ da concentração deste causou um aumento de incorporação de $100 \%$. Da mesma maneira para uma mesma concentração de hidrogênio observou-se um aumento do índice de fluidez do polímero com conseqüente diminuição do peso molecular. Tal efeito está de acordo com o esperado uma vez que o comonômero também atua como agente de transferência de cadeia, favorecendo um aumento da constante de transferência para este. Assim ocorre um decréscimo do peso molecular uma vez que este é determinado pela relação entre a constante de propagação e a constante de transferência de cadeia $\left(\mathrm{k}_{\mathrm{p}} / \mathrm{k}_{\mathrm{tr}}\right)$.

\section{Conclusões}

Pela análise dos catalisadores Ziegler-Natta mistos pode-se concluir que o composto de metaloceno está presente no catalisador final. A análise de titânio por via úmida mostrou um aumento do percentual de titânio proporcional à quantidade de metaloceno utilizada.

O catalisador metalocênico sólido $\mathrm{CpTiCl}_{3}$, por meio do tratamento com uma solução de triisobutilalumínio (TIBA) e formação do composto $(\mathrm{Cp})(\mathrm{iBu}) \mathrm{TiCl}_{2}$ solúvel no meio pode ser fixado nas faces do $\mathrm{MgCl}_{2}$ do catalisador Ziegler-Natta heterogêneo. A fixação do novo composto de titânio ligado a um anel ciclopentadienílico introduz um novo tipo de centro ativo no catalisador Ziegler-Natta, conforme proposto neste trabalho, capaz de modificar o desempenho do mesmo na polimerização de olefinas. $\mathrm{O}$ novo tipo de centro ativo $\mathrm{CpTiCl}_{2}(\mathrm{iBu})$ fixado na face (100) do $\mathrm{MgCl}_{2}$ do catalisador ZieglerNatta base, já apresenta um grupo alquila e uma vacância, o que justifica a sua atividade em menor concentração de cocatalisador.

Os catalisadores mistos se mostraram ativos tanto para a produção de PEAD como de PELBD. Em ambos os casos, percebe-se que a atividade variou com a razão molar $\mathrm{Al} / \mathrm{Ti}$ e que para valores inferiores a 500 não se observou polimerização de etileno. Entretanto, para copolimerizações de etileno e 1-buteno, o catalisador é ativo mesmo em valores de razões molares de Al/Ti tão baixos quanto 60 .

A concentração de alquilalumínio necessária para se conseguir valores de atividades aceitáveis com catalisadores mistos em copolimerização de etileno1-buteno é relativamente baixa, o que representa uma vantagem econômica do sistema estudado.

\section{Referências Bibliográficas:}

1. Andresen, A.; Cordes, H.G.; Herwing, J.; Kaminsky, W.; Merk, A.; Mottweiler, R.; Penn, J.; Sinn, H.; Vollmer, H.J. - Angew. Chem., 88, p.689 (1976).

2. Andresen, A.; Cordes, H.G.; Herwing, J.; Kaminsky, W.; Merk, A.; Mottweiler,R.; Penn, J.; Sinn, H.; Vollmer, H.J. - Angew. Chem. Int. Ed. Engl., 15, p.630 (1976).

3. Ewen, J.A. - J.Am. Chem. Soc., 106, p.6355 (1984).

4. Ahlers, A. - Kaminsky, W. - Makromol. Chem. Rapid Commun., 9, p.457 (1988).

5. Cho, H.S.; Chung, J.S.; Han, J.H.; Ko, Y.G.; Lee, W.Y. - J. Appl. Polym. Sci.,70, p.1707 (1998).

6. Leaversuch, R. - Modern Plastics International, p.38 (1994).

7. Alves, O. L. - Química Nova, 9, p.276 (1986).

8. A.I. Vogel, Análise Inorgânica Quantitativa, 4ed. RJ: Guanabara (1981).

9. Pizzol, M.F.D. - “Estudo das Estruturas de Polietilenos Lineares de Baixa Densidade Obtidos com Catalisadores Ziegler-Natta e Metaloceno", Dissertação de Mestrado, Universidade Federal do Rio Grande do Sul, Brasil (1997).

10. Barbé, P.C.; Cecchin, G.; Noristi, L. - Adv. Polym. Sci., 81, p.1 (1987).

11. Corradini, P.; Villani, V.; Guerra, G. - Macromolecules, 18, p.1041 (1985).

12. Busico, V.; Corradini, P.; Martino, L.; Proto, A.; Savino, V. - Makromol. Chem., 186, p.1279 (1985).

13. Busico,V.; Cipullo, R.; Corradini, P.; Biasio, R.D. Makromol. Chem. Phys., 196, p.491 (1995).

14. Forte, M.C.; Coutinho, FM.B. - Eur. Polym. Journal, 32, p.223 (1996).

15. K. Hasebe; H. Mori; M. Terano; -J. Mol. Catal. A:Chem., 124, p.L1, (1997)

16. K. Hasebe; H. Mori; M. Terano; - J. Mol. Catal. A:Chem., 140, p.165, (1999).

17. K., Hasebe; H., Mori; M. Terano; -Polym. 40 p.1389, (1999).

18.E. Magni; G.A. Somorjai; - J. Phys. Chem., 102 p. 8788, (1998).

19. A.Harlin, PCT WO 96/18677 Borealis Polymers OY (1994).

20. P.P.Shirodkar, European Patent Application 0528523A1, Mobil Oil Corporation,(1992)

21. Strate, G.V.; Cozewith, C.; Ju, S. - Macromolecules, 21, p.3360 (1988).

Recebido: $10 / 02 / 00$

Aprovado: 24/08/00

Polímeros: Ciência e Tecnologia, vol. 10, nº 3, p. 122-129, 2000 